\title{
Evaluation protocol before, during, and after bronchoscopy-guided swallowing test for decannulation: clinical audit proposal
}

This article was published in the following Dove Press journal:

Clinical Audit

\author{
Olivia Sánchez-Cabral' \\ Patricio Santillán-Doherty ${ }^{2}$ \\ Dina Martínez-Mendoza ${ }^{1,3}$ \\ 'Interventional Pulmology Unit, \\ ${ }^{2}$ Medical Direction, ${ }^{3} \mathrm{Hospital}$ \\ Epidemiology Surveillance Unit, \\ National Institute of Respiratory \\ Diseases Ismael Cosío Villegas, \\ Mexico City, Mexico
}

\begin{abstract}
Swallowing is a complex act. In some series, it was found that $\sim 20 \%$ of patients under invasive mechanical ventilation in intensive care units are subjected to percutaneous tracheostomy; however, the presence of tracheostomy causes an increase in the amount of tracheal secretions, changes in the deglutition mechanism, a higher risk of airway infection, bleeding, and hinders vocalization, causing late complications. Decannulation must be performed as soon as possible, since it facilitates phonation and deglutition. This project presents the methods for undertaking a clinical audit to evaluate patients with tracheostomy before, during, and after bronchoscopy-guided swallowing test for decannulation purposes. The main goal is to evaluate the effectiveness of decannulation in patients with percutaneous tracheostomy through physiological and anatomical examination of the airway and the swallowing mechanism using the bronchoscopic method. This method consists of a series of the following evaluations: 1) select patient candidate for swallowing test; 2) prior preparation of the patient for the swallowing test; and 3) transnasal phase, which decides if the patient should be decannulated, consisting of evaluation of the bronchoscopy-guided swallowing test. The patient should not have alterations. If he/she has no alterations in the transnasal and/or transtracheostomy phases, the patient is decannulated. A reliable procedure will be performed by the study data collectors. Data will be analyzed according to the nature of the variables. Decannulation success will be determined by inferential statistics.
\end{abstract}

Keywords: swallowing test, decannulation, bronchoscopy-guided evaluation, interventional pulmonology

\section{Background}

Swallowing is a complex act involving four phases, the preoperative, oral, pharyngeal, and esophageal phases. Sensory and motor integrity as well as an adequate coordination and the sequential functioning of all four phases are required for normal swallowing function. ${ }^{1}$ The preoperative and oral phases involve mastication and bolus transference and the pharyngeal phase requires an adequate velopharyngeal, lingual, hypopharyngeal, and laryngeal function. In the esophageal phase, the upper esophageal sphincter is relaxed and the peristaltic waves begin. ${ }^{2,3}$

In some series, it was found that $\sim 20 \%$ of patients under invasive mechanical ventilation at the Intensive Care Units are subjected to percutaneous tracheostomy. ${ }^{4}$ This procedure is indicated when an upper airway obstruction occurs, in order to improve comfort and to enable a safe airway. It also facilitates ventilator removal, reducing the rate of tracheolaryngeal complications caused by the prolonged permanence of the
Correspondence: Dina Martínez-Mendoz Hospital Epidemiology Surveillance Unit. National Institute of Respiratory Diseases Ismael Cosío Villegas, Calzadade Tlalpan 4502, Col Sección XVI, Mexico City 14080, Mexico Email dinamarty@gmail.com 
orotracheal tube. ${ }^{5}$ However, tracheostomy causes an increase in the amount of tracheal secretions, changes in the deglutition mechanism, increase in the risk of airway infection, bleeding, and hinders vocalization. ${ }^{6-8}$ Late complications are diagnosed in $65 \%$ of patients. Granuloma is the most frequent complication, followed by structural injury such as malacia, stenosis, and fistulae. ${ }^{9,10}$ In previous studies, this occurred in up to $67 \%$ of patients. ${ }^{11,12}$ Decannulation must be carried out as soon as possible to avoid these complications and improve the patient's comfort and physical appearance, as it facilitates phonation and deglutition.

Decannulation failure is characterized by the need for reintubation within 48-96 hours after decannulation is initially performed. This can occur in $5 \%$ of patients. ${ }^{8,13} \mathrm{~A}$ multidisciplinary approach including swallowing evaluation may lead to a high percentage of decannulation, even more rapidly in comparison to an approach lacking a multidisciplinary protocol. ${ }^{11}$

The association between the presence of a tracheostomy tube and bronchoaspiration is due to a limited elevation of the pharynx, diminished pharyngeal sensitivity, and an increase in subglottic pressure as well as poor cough reflex, ${ }^{14}$ although the aim of a tracheostomy cannula cuff is to provide an occlusion around the tube, facilitating ventilation and reducing the passage of secretions. The studies performed have not been able to demonstrate changes in aspiration levels between inflated and deflated cuff. ${ }^{13,15}$ Up to $30 \%-40 \%$ of silent aspirations are not detected in clinical evaluations. ${ }^{16,17}$

Other decannulation protocols have been proposed to reduce the diameter of tracheostomy cannulae, but it has been demonstrated that a smaller diameter results in an increase of diaphragmatic effort, a reduction in tidal volume, and an increase in intrinsic positive end expiratory pressure. ${ }^{18,19}$

The importance of maintaining the tracheostomy tube cuff, inflated or deflated in decannulation, was analyzed in a series of 17 patients with the presence of tracheostomy cuffs. Thirteen $(76 \%)$ were able to keep the cuff deflated and four (24\%) were not able to keep it. Out of the patients who could keep the deflated cuff, two (15\%) could not be decannulated for other reasons. However, 11 (64.7\%) were able to initiate the decannulation process. This demonstrates that keeping the cuff deflated is an important decannulation predictive factor. ${ }^{20}$

The occlusion test tolerance capacity was assessed in an epidemiological study by Stelfox et al. ${ }^{13}$ They found that the capacity to tolerate this test is related to sensitivity and low specificity, since some patients who did not tolerate occlusion were successfully decannulated and, in contrast, most patients were decannulated without using this occlusion test.
The method of gradual tube downsizing has been used as a decannulation protocol. However, it has been questioned because resistance is inversely proportional to the tube's radius. This reduction of the tube's radius leaves the patient exposed to increased resistance. ${ }^{21-23}$

Bronchoscopy-guided swallowing test includes the secretion assessment and/or food stagnation in the pyriform sinuses, which may be caused by a lack of pharyngeal sensitivity, and/or motility, or by cricopharyngeal muscle spasms. The presence of gastroesophageal laryngopharyngeal reflux may be revealed by the presence of posterior glottic commissure edema, or by an arytenoid granuloma, and/or by direct visualization of reflux following deglutition. The postcricoid region can be visualized by the Valsalva maneuver. This test is usually performed using different food consistencies (solid, semi-liquid, and liquid), naturally colored, or by adding some test dye drops, such as methylene blue, to distinguish the laryngopharyngeal mucosa and the natural oropharyngeal secretions. $^{24}$

The bronchoscopy-guided swallowing exam has shown adverse effects such as discomfort, nausea, vomiting, vasovagal response, changes in heart rate, syncope, epistaxis, and laryngospasm that are minimal and insignificant. A study of 500 patient evaluations with various underlying diagnoses revealed minor epistaxis which subsided spontaneously in $0.6 \%$ and no incidences of laryngospasm or vasovagal response were found. ${ }^{25,26}$

Chronic comorbidities and failure of evidence-based decannulation guidelines hinder the prediction of decannulation outcomes in individual patients. ${ }^{27}$

\section{Methods}

This clinical audit shall be performed at the Interventionist Pneumology Unit of the National Institute of Respiratory Diseases Ismael Cosío Villegas of Mexico and shall take place between September 1, 2018 and August 31, 2019. The study variables include sociodemographic data and clinical data, and the evaluation results will be shown in accordance with the registration cards at the clinical auditing, as well as the informed consent duly signed by the patient.

The audit is a proposal based on a cohort study perspective of the objectives, which has been approved by the Research and Ethics Committees of the National Institute of Respiratory Diseases Ismael Cosío Villegas of Mexico with code number C06-16.

The general objective of the instant audit is to assess the decannulation effectiveness in percutaneous tracheostomy patients through anatomical and physiological examination 
of the airway and the swallowing mechanism, using the bronchoscopic method.

The specific objectives of this study are as follows:

- to assess the success rate in the patient candidate: patients who have criteria to be evaluated through a swallowing test, then, we consider the meaning is correct;

- to assess the decannulation success rate in patients not showing any disorders at the tracheostomy phase, during the swallowing test, and who can continue to the transnasal evaluation phase;

- to assess the decannulation success rate in patients who did not show any disorders in the swallowing test, as well as in the tracheostomy phase and in the transnasal phase;

- to identify the most frequent variables in patients who are not candidates for the swallowing test; and

- to identify the factors related to the decannulation contraindications in any of the test phases.

For the clinical audit, we designed our own specific data collection method, which consisted of flowcharts showing results, in a sequential manner, beginning from the time of the patient arrival to the performance of the swallowing test with the objective of decannulation.
We also designed evaluation cards to follow all the variables considered at the clinical and bronchoscopic approach phase. The scales used in this study are widely known and are duly referenced in this paper.

The clinical audit aims to measure the effectiveness of decannulation by way of the bronchoscopy-guided swallowing test protocol results. It consists of performing a series of evaluations as follows: 1) selection of swallowing test candidate patients; 2) patient preparation for the swallowing test; and 3) evaluation of the bronchoscopy-guided swallowing test. The procedure consists of two phases: 1) the transtracheostomy phase and 2) the transnasal phase. Alterations shall not be present in candidates for decannulation (Figure 1).

\section{Study population}

Patients $\geq 18$ years of age with previous percutaneous tracheostomies carried out at an Intensive Care Unit or performed during this study period were included.

\section{Selection criteria}

Patients are selected by way of an evaluation card shown in Figure 2, where the criteria for a patient to become a candidate for the swallowing test are described at the evaluation previous to the swallowing test.

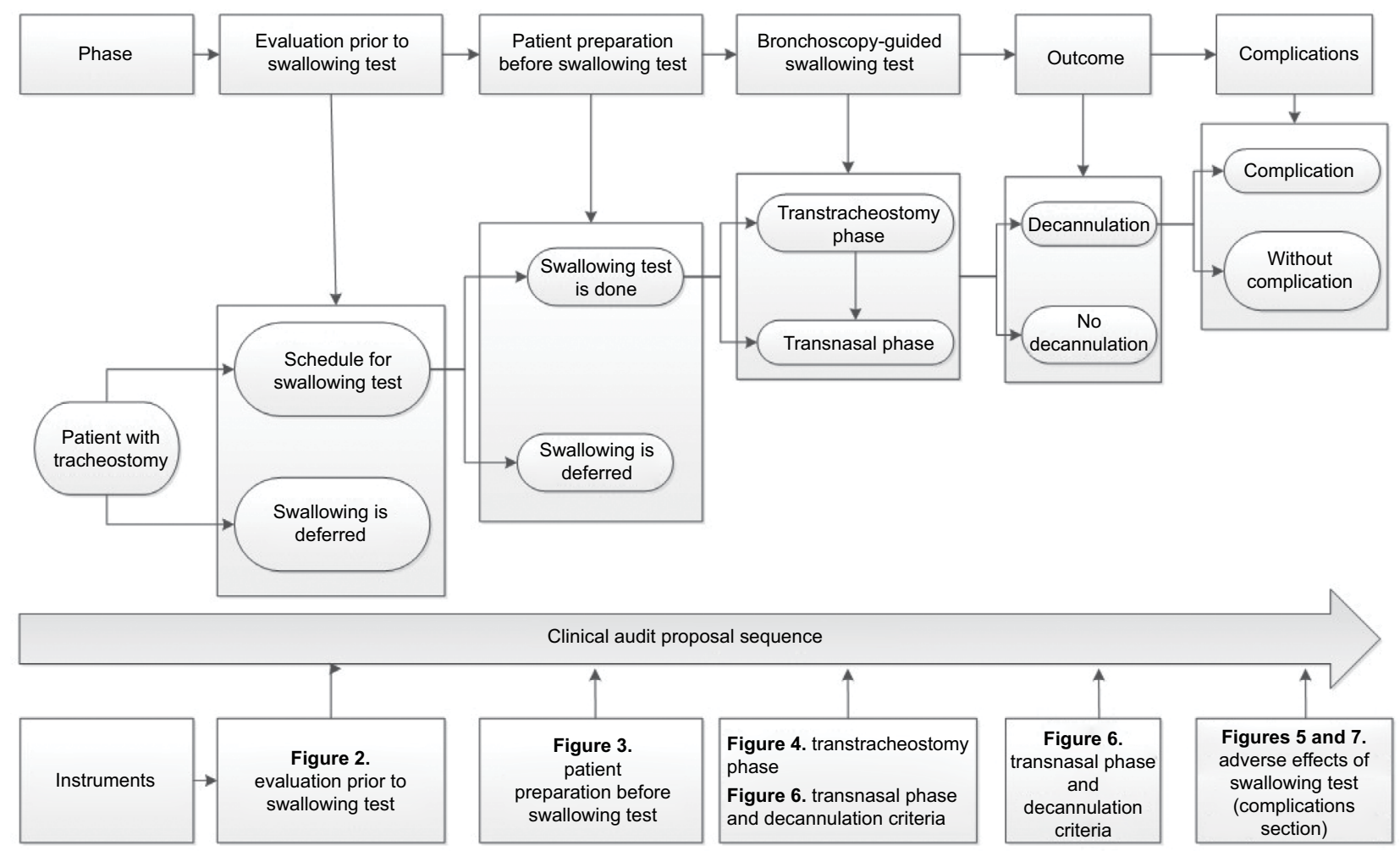

Figure I Clinical audit proposal: evaluation protocol before, during, and after bronchoscopy-guided swallowing test for decannulation purposes. 


\begin{tabular}{|c|c|}
\hline Yes & Conditions \\
\hline $\mathrm{O}$ & Absence of airway obstruction? \\
\hline $\mathrm{O}$ & Has the indication for mechanical ventilation been resolved or is in resolution? \\
\hline $\mathrm{O}$ & Is the patient without mechanical ventilatory support? \\
\hline $\mathrm{O}$ & Does the patient have previous swallowing disorder? \\
\hline $\mathrm{O}$ & Does the patient eat? And if so, he feeds himself or with assistance? \\
\hline $\mathrm{O}$ & Does the patient mobilize himself in bed and/or is ambulatory? \\
\hline $\mathrm{O}$ & Does the patient need aspiration less than two times every 8 hours? \\
\hline- & Glasgow scale: \\
\hline$\overline{\mathrm{O}}$ & Glasgow $\geq 9$ ? \\
\hline- & Karnofsky scale: ___ \\
\hline$\overline{\mathrm{O}}$ & Karnofsky $>50 ?$ \\
\hline $\mathrm{O}$ & Effective cough reflex? \\
\hline $\mathrm{O}$ & $\mathrm{SO}_{2} \geq 92 \%$, either at ambient air or with supplemental $\mathrm{O}_{2}$ ? \\
\hline $\mathrm{O}$ & $\mathrm{PaO}_{2} / \mathrm{FIO}_{2}$ ratio $>150 \mathrm{mmHg}$ \\
\hline $\mathrm{O}$ & Absence of noncompensated respiratory and/or metabolic acidosis \\
\hline $\mathrm{O}$ & $\begin{array}{l}\text { Presence of swallowing reflex? (motor examination with larynx palpation, with capacity to swallow secretions, } \\
\text { spontaneous swallowing more than one per minute) }\end{array}$ \\
\hline $\mathrm{O}$ & Does the patient handle oropharyngeal secretions? Accumulation of abundant saliva in the larynx \\
\hline $\mathrm{O}$ & Negative bronchial secretion cultures or covered by specific antibiotics? \\
\hline $\mathrm{O}$ & ECG without signs of recent myocardial ischemia \\
\hline $\mathrm{O}$ & No need for vasoactive drugs \\
\hline $\mathrm{O}$ & Heart rate $<140$ beats $/ \mathrm{min}$ \\
\hline $\mathrm{O}$ & Hemoglobin $>8 \mathrm{~g} / \mathrm{dL}$ \\
\hline $\mathrm{O}$ & Temperature $<38^{\circ} \mathrm{C}$ \\
\hline $\mathrm{O}$ & Capacity to maintain sitting position \\
\hline
\end{tabular}

Figure 2 Evaluation prior to swallowing test.

Note: If any of these conditions are not present, the swallowing test will be deferred.

Abbreviations: ECG, electrocardiogram; $\mathrm{PaO}_{2} / \mathrm{FIO}_{2}$, ratio of arterial oxygen partial pressure to fractional inspired oxygen.

\section{Evaluation prior to swallowing test}

The evaluation prior to swallowing test is described in Figure 2.

For a patient to be considered as a candidate for the swallowing test, the following factors/conditions should be met. Upper airway obstruction should be absent in the patient. The patient should be in recovery phase or the pathology that led to mechanical ventilation should have been previously resolved. The patient should not be subjected to mechanical ventilatory support. The patient should not show previous deglutition disorders. The patient should be able to feed himself or with assistance. The patient should be mobile in bed and/or able to walk. Aspiration should be limited to less than two times every 8 hours. The patient should have effective cough reflex. Deglutition reflex should be present (motor examination with larynx palpation, the capacity to swallow secretions, spontaneous deglutition more than one per minute). The patient must have normal control of oropharyngeal secretions (without accumulation of saliva in the larynx). There should be a negative bronchial secretion culture or he/she should have been treated with specific antibiotics.
The patient's state of consciousness should be in accordance with the Glasgow scale $\geq 9$, Karnofsky scale $>50, \mathrm{SO}_{2} \geq 92 \%$, either with ambient air or with supplemental oxygen, and ratio of arterial oxygen partial pressure to fractional inspired oxygen $>150 \mathrm{mmHg}$. Noncompensated respiration and/or metabolic acidosis should be absent. Chest X-ray should be without abnormalities or show an improvement compared to previous respiratory pathology. The electrocardiogram should not show signs of recent myocardial ischemia. No need for vasoactive drug use, heart rate $<140$ beats $/ \mathrm{min}$, hemoglobin $>8 \mathrm{~g} / \mathrm{dL}$, and temperature $<38^{\circ} \mathrm{C}$. The patient should be able to maintain an upright position. If any of these conditions are not met, the swallowing test will be deferred..$^{11,13,16,28-31}$

\section{Patient preparation before swallowing test}

Figure 3 shows the patient preparation before the swallowing test.

Patient preparation for performance of the swallowing test consists of a 3-5 day special diet prior to the swallowing test, as well as deglutition exercises, and specific insufflation 


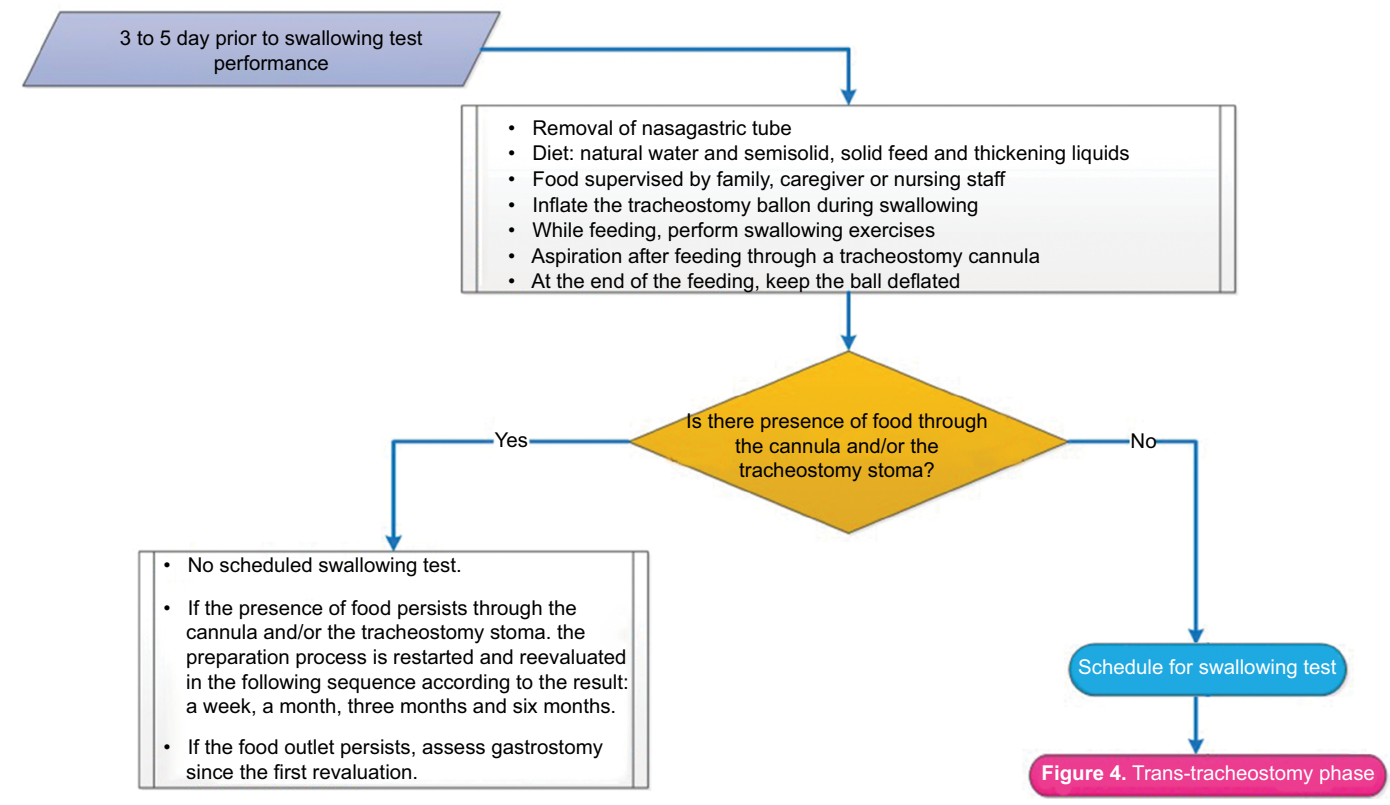

Figure 3 Patient preparation before swallowing test.

of the tracheostomy cannula cuff. At the end of preparation, it is decided whether to schedule the swallowing test or to reevaluate the patient in a week's time.

Once the patient is prepared for the swallowing test, the following foods should be available during the test phase: First liquids: $50 \mathrm{~mL}$ of juice. Grape or blackberry juice is preferred due to their color since it facilitates visualization of medium penetration. Then, semi-solid foods: $100 \mathrm{~g}$ of baby food consistency Greek yogurt. Finally, "Marie wafer"-type cookies are used as solid food due to their low fat and sugar content (7.5 g [two cookies]).

\section{Evaluation of the bronchoscopy-guided swallowing test}

The procedure consists of two phases: the transtracheostomy phase and the transnasal phase. To decide decannulation, the patient should not have alterations.

Prior to the procedure, local anesthesia consisting of 5 $\mathrm{mL}$ of lidocaine $1 \%$ plain is administered in the tracheostomy stoma, nasal pits, and larynx.

The bronchoscopy procedure is performed with an Olympus fibro-bronchoscope model IT160.

\section{Transtracheostomy phase}

Figures 4 and 5 show the transtracheostomy phase.

With the patient in a sitting position in at least $70^{\circ}$, the tracheostomy cannula is removed to facilitate exploration as well as to assess forced inspiration and exhalation; asepsis and antisepsis of the tracheostomy stoma site is performed. The endoscope tip is then directed upward toward the subglottic region with retroflexion movement, showing the inferior surface of the vocal folds. The glottis appears in reverse position compared to the image obtained by transnasal or transoral laryngeal endoscopy. Such a position facilitates visualization of the stoma, movements of the vocal folds, and evaluation of secretion and/or food deglutition in the trachea during the predeglutition, transdeglutition, and postdeglutition phases. ${ }^{14,25,32}$

The swallowing test consists of food intake previously described by the patient in the above-stated sequence, that is, liquid, semi-solid, and solid. Bronchoaspiration is evaluated at each step. Bronchoaspiration is defined as the passage of any of the aforementioned foods under the vocal folds into the trachea. If massive spontaneous bronchoaspiration of oropharyngeal secretions determined by the Donzelli scale grade 4 or food Penetration-Aspiration scale score $>4$ occurs, the test is interrupted.

\section{Transnasal phase}

Figures 6 and 7 show the transnasal phase.

The fibro-bronchoscope is placed in two positions, in an upper position near the velum palatinum ahead of the epiglottis during deglutition and then the same is moved forward to a lower position at the level of the laryngeal vestibule. ${ }^{14,25}$ 


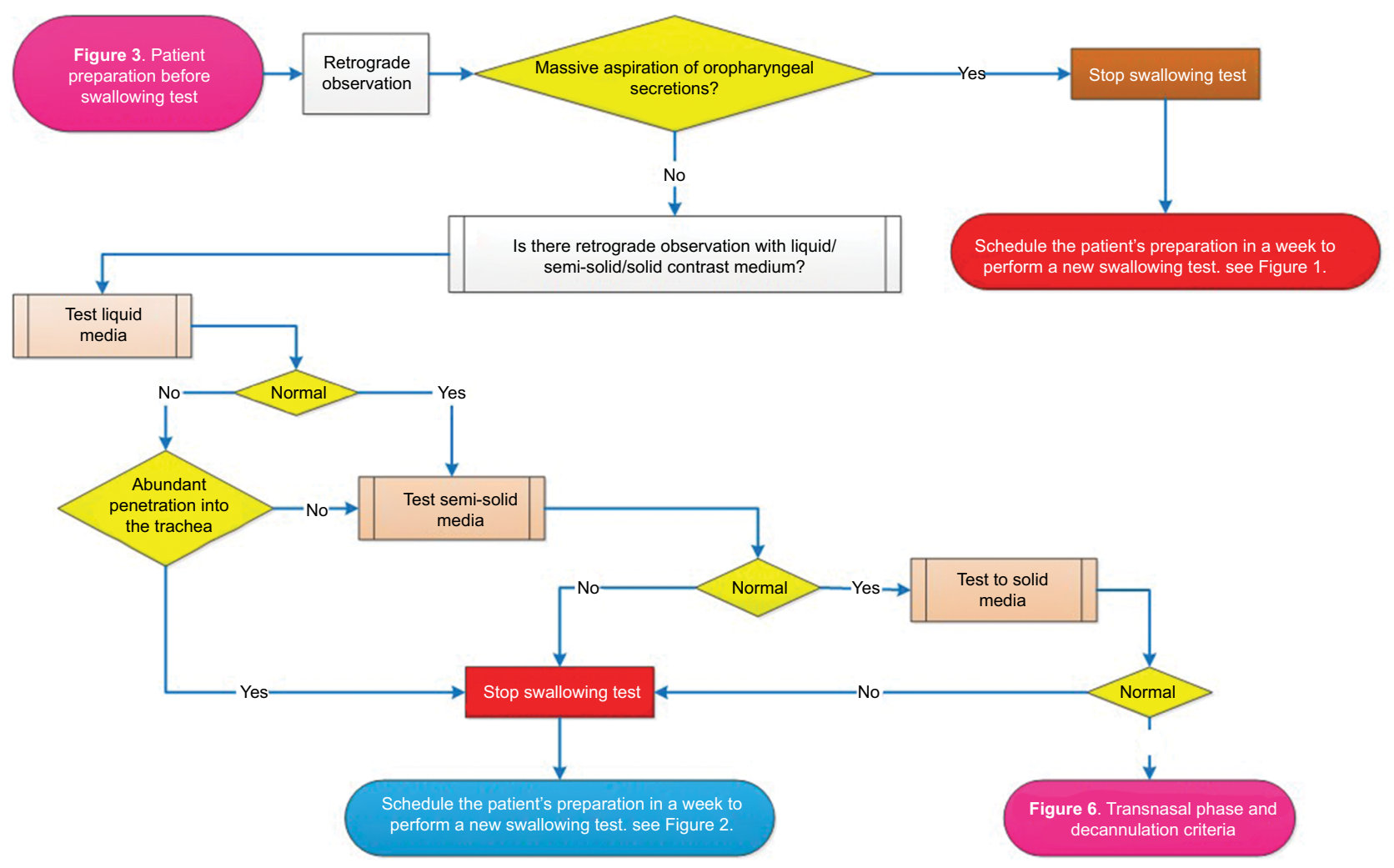

Figure 4 Transtracheostomy phase.

The cough reflex is explored by assessing the laryngeal and pharyngeal sensitivity, touching the mucosa with the endoscope tip. ${ }^{26}$

Soft palate function is evaluated during phonation and deglutition, assessing the possible presence of velopharyngeal insufficiency, which causes rhinophonia, rhinolalia aperta, and nasal food reflux (particularly liquid).

Laryngopharyngeal function is assessed during phonation, showing the possible presence of vocal cord palsy. ${ }^{24}$

The severity of secretion retention is recorded using a modified Donzelli scale, ${ }^{33}$ from grade 1 (scarce secretions occupying $<10 \%$ of pyriform sinuses and valleculae) to grade 4 (manifest secretion aspiration).

Endoscopic swallowing evaluation allows assessment of the deglutition pharyngeal phase. It only shows the pre- and postdeglutition phases. The glottic closure during intradeglutition phase is not adequately shown, since it is covered by elevation of the larynx with subsequent rotation of the epiglottis by the posterior movement of the base of tongue and contraction of the pharyngeal walls. ${ }^{34}$

The Cotton classification is used to assess tracheal stenosis, ${ }^{35}$ while the Expiratory Central Airway Collapse classification evaluates the functioning, extent, and severity of the collapse (in percentage) of the central airway. This is used to assess tracheobronchomalacia. ${ }^{36-38}$

\section{Decannulation criteria}

Figure 6 shows the decannulation criteria.

If no alterations are found in the transnasal and/or transtracheostomy phases, the patient is decannulated. Decannulation is contraindicated in the following circumstances: lesion of bilateral vocal cords in adduction, subglottic obstruction or lesion $>50 \%$ or grade II or higher obstruction according to Cotton classification, abundant peristomal granulation tissue $>50 \%$ from the lumen that cannot be resected, grade 4 tracheobronchomalacia, grade 4 Expiratory Central Airway Collapse classification, spontaneous massive bronchoaspiration of oropharyngeal secretions according to the Donzelli scale, or a score $>4$ in the food Penetration-Aspiration scale; regarding functioning, absence of cough reflex, no secretion clearing after three deglutitions, chordal palsy, and deglutition disorders with the various media. ${ }^{39}$

Placement of phonation valves allows the patient to speak, increasing motivation and accelerating recovery. ${ }^{40}$ 


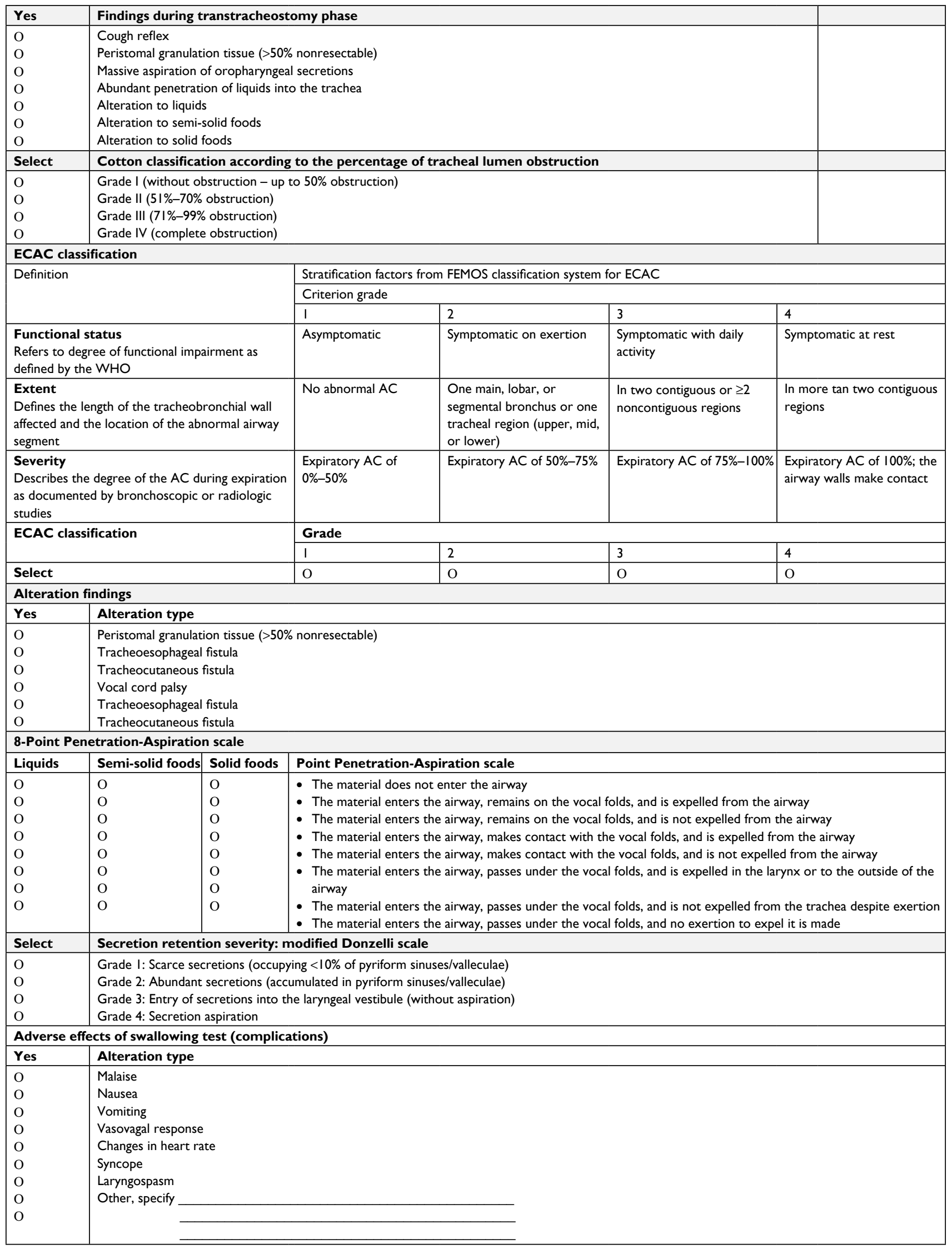

Figure 5 Transtracheostomy phase.

Abbreviations: AC, airway collapse; ECAC, Expiratory Central Airway Collapse; FEMOS, The multidimensional classification system Functional status, Extent, Morfology, Origin and Severity of the abnormalities; WHO, World Health Organization. 


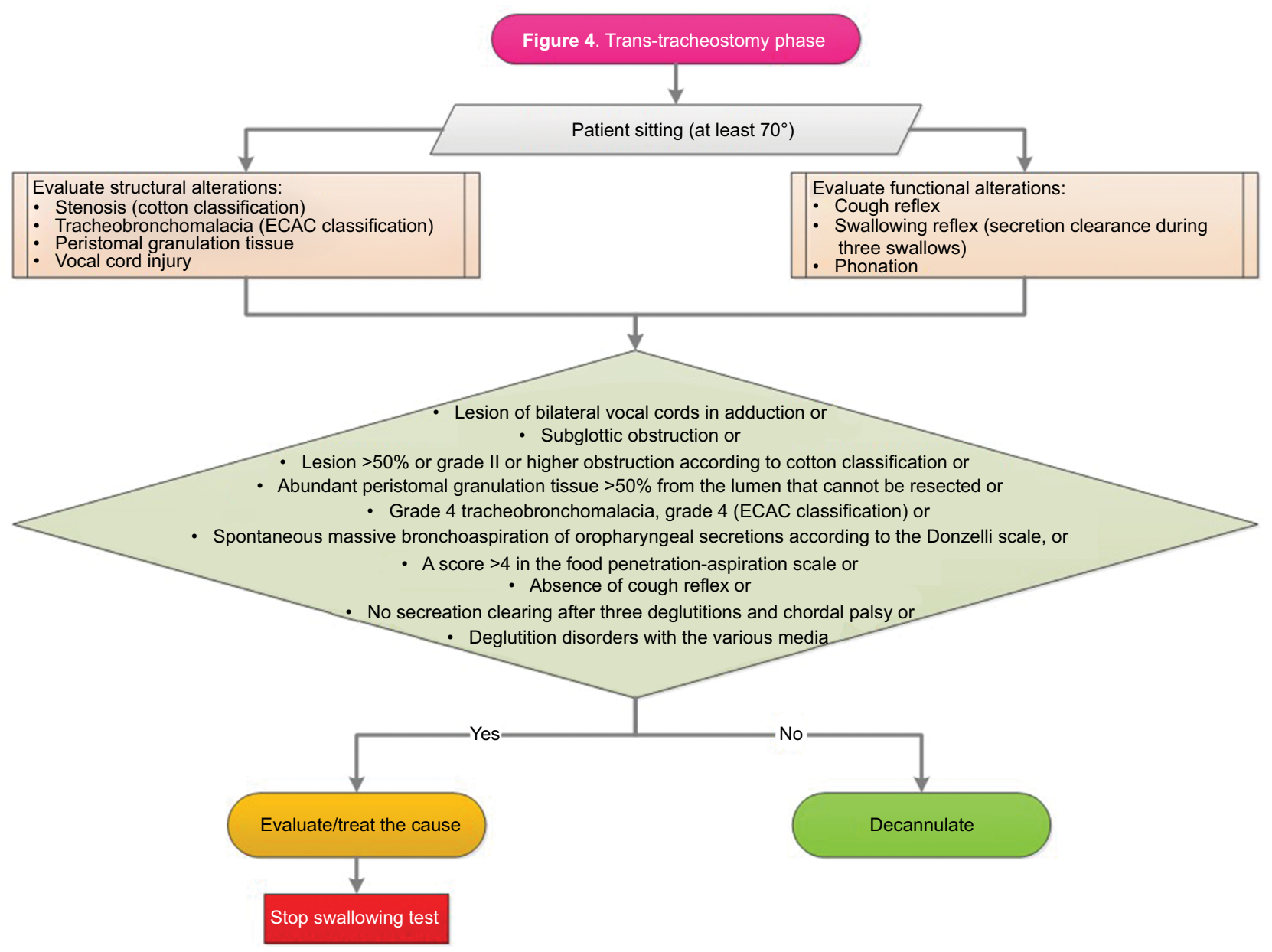

Figure 6 Transnasal phase and decannulation criteria.

Abbreviation: ECAC, Expiratory Central Airway Collapse.

When the swallowing test shows alterations in any of its two phases, decannulation is not performed and the patient is prepared within 1 week for a repeat of swallowing test. In case of continuous alterations, the patient is scheduled for another swallowing test in 1 month. A gastrostomy probe is placed and fenestrated cannula with phonation valve is relocated, so that a subglottic aspiration can be performed. Deglutition exercises are continued and follow-up rehabilitation is carried out by a therapist for a period of 3 months before rescheduling a swallowing test.

The assessments shall be carried out by an experienced interventionist pulmonologist.

\section{Analysis plan}

In each and all of the sociodemographic clinical and bronchoscopic variables, descriptive statistics will be used in accordance with the nature of the variable. The success rate of the decannulation will be calculated in the different corresponding test populations: 1) decannulation success rate in patients who are candidates for the swallowing test; 2) decannulation success rate in patients who did not show any alteration at the transtracheostomy phase during the swallowing test and moved into the transnasal phase of the bronchoscopic test; and 3) decannulation success rate in patients who did not show any alteration in the swallowing test at the transtracheostomy phase and the transnasal phase.

Identify the most frequent variable found in patients who are not candidates for the swallowing test. To identify the associated factors and contraindication of decannulation in any one of the test phases, compare the population with and without decannulation by way of chi-squared test or Fisher's test, where it may be applied based on the different categories and the differences in measurement with the Student's $t$-test for numbering variables. $P$-values $<0.05$ will be considered statistically significant. The statistical package IBM SPSS 21 will be used for the analysis. 


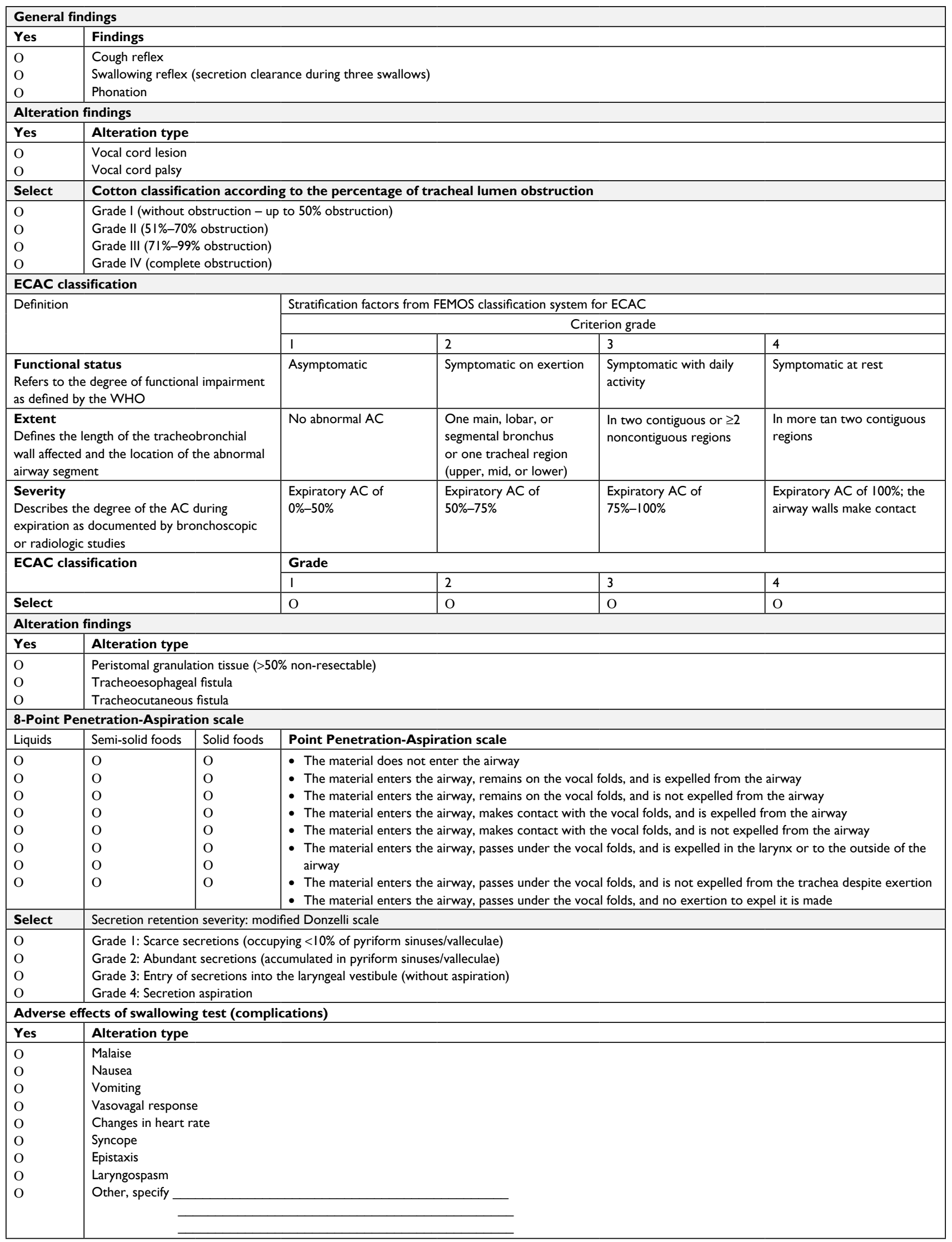

Figure 7 Transnasal phase.

Abbreviations: ECAC, Expiratory Central Airway Collapse; FEMOS, The multidimensional classification system Functional status, Extent, Morfology, Origin and Severity of the abnormalities; WHO, World Health Organization. 


\section{Disclosure}

The authors report no conflicts of interest in this work.

\section{References}

1. Garuti G, Reverberi C, Briganti A, Massobrio M, Lombardi F, Lusuardi M. Swallowing disorders in tracheostomised patients: a multidisciplinary/multiprofessional approach in decannulation protocols. Multidiscip Respir Med. 2014;9(1):36.

2. Richard I, Giraud M, Perrouin B. Ciance D. Laryngotracheal stenosis after intubation or tracheostomy in patient with neurological disease. Arch Phis Med Rehabil. 1993;74:905-909.

3. Lombardi F, Andreoli E, Dardani M, Vezzosi G, Brianti R. Gestione integrata della disfagia e della cannula tracheostomica nel grave cerebroleso in Riabilitazione. Giornale Italiano di Medicina Riabilitativa. 2003; 17:87-95.

4. Esteban A, Anzueto A, Alía I, et al. How is mechanical ventilation employed in the intensive care unit? An international utilization review. Am J Respir Crit Care Med. 2000;161(5):1450-1458.

5. Castella X, Gilabert J, Torner F. Laryngeal damage from intubation. Chest. 1990;98(3):776-777.

6. Romero CM, Marambio A, Larrondo J, et al. Swallowing dysfunction in nonneurologic critically ill patients who require percutaneous dilatational tracheostomy. Chest. 2010;137(6):1278-1282.

7. Heffner JE. Tracheostomy decannulation: marathons and finish lines. Crit Care. 2008;12(2):128.

8. Ceriana P, Carlucci A, Navalesi P, et al. Weaning from tracheotomy in long-term mechanically ventilated patients: feasibility of a decisional flowchart and clinical outcome. Intensive Care Med. 2003;29(5):845-848.

9. Epstein SK. Late complications of tracheostomy. Respir Care. 2005;50(4):542-549.

10. Grillo HC. Surgery of the Trachea and Bronchi. Hamilton: BC Decker; 2004.

11. Maruvala S, Chandrashekhar R, Rajput R. Tracheostomy decannulation: when and how? Res Otolaryngol. 2015;4(1):1-6.

12. Law JH, Barnhart K, Rowlett W, de La Rocha O, Lowenberg S. Increased frequency of obstructive airway abnormalities with long-term tracheostomy. Chest. 1993;104(1):136-138.

13. Stelfox HT, Crimi C, Berra L, et al. Determinants of tracheostomy decannulation: an international survey. Crit Care. 2008;12(1):R26.

14. Brady SL, Wesling M, Donzelli J. Pilot date on swallow function in nondysphagic patients requiring a tracheotomy tube. Int J Otolaryngol. 2009;2009:610849-4.

15. Santus P, Gramegna A, Radovanovic D, et al. A systematic review on tracheostomy decannulation: a proposal of a quantitative semiquantitative clinical score. BMC Pulm Med. 2014;14:201.

16. Hales PA, Drinnan MJ, Wilson JA. The added value of fibreoptic endoscopic evaluation of swallowing in tracheostomy weaning. Clin Otolaryngol. 2008;33(4):319-324.

17. Linden P, Kuhlemeier KV, Patterson C. The probability of correctly predicting subglottic penetration from clinical observations. Dysphagia. 1993;8(3):170-179.

18. Valentini I, Tonveronachi E, Gregoretti C, et al. Different tracheotomy tube diameters influence diaphragmatic effort and indices of weanability in difficult to wean patients. Respir Care. 2012-2018;57.

19. Criner G, Make B, Celli B. Respiratory muscle dysfunction secondary to chronic tracheostomy tube placement. Chest. 1987;91(1):139-141.
20. Zanata IL, Santos RS, Hirata GC. Tracheal decannulation protocol in patients affected by traumatic brain injury. Int Arch Otorhinolaryngol. 2014;18(2):108-114.

21. Cohen O, Tzelnick S, Lahav Y, et al. Feasibility of a single-stage tracheostomy decannulation protocol with endoscopy in adult patients. Laryngoscope. 2016;126(9):2057-2062.

22. Lewarski JS. Long-term care of the patient with a tracheostomy. Respir Care. 2005;50:534-537.

23. Wasserzug O, Adi N, Cavel O, et al. One-stage decannulation procedure for patients undergoing oral and oropharyngeal oncological surgeries and prophylactic tracheotomy. Open Otorhinolaryngol J. 2010;4(1):73-76.

24. Langmore SE, Schatz K, Olsen N. Fiberoptic endoscopic examination of swallowing safety: a new procedure. Dysphagia. 1988;2(4): 216-219.

25. Nacci A, Ursino F, La Vela R, Matteucci F, Mallardi V, Fattori B. Fiberoptic endoscopic evaluation of swallowing (FEES): proposal for informed consent. Acta Otorhinolaryngol Ital. 2008;28(4):206-211.

26. Aviv JE, Kaplan ST, Thomson JE, Spitzer J, Diamond B, Close LG. The safety of flexible endoscopic evaluation of swallowing with sensory testing (FEESST): an analysis of 500 consecutive evaluations. Dysphagia. 2000;15(1):39-44.

27. Nicolini A, Piroddi IMG, Banfi P. Tracheostomy decannulation. Phys Med Rehabil Int. 2015;2(6):1053.

28. Marchese S, Corrado A, Scala R, Corrao S, Ambrosino N; Intensive Care Study Group, Italian Association of Hospital Pulmonologists (AIPO). Tracheostomy in patients with long-term mechanical ventilation: a survey. Respir Med. 2010;104:749-753.

29. Heffner JE. The technique of weaning from tracheostomy. Criteria for weaning; practical measures to prevent failure. J Crit Illn . 1995;10(10):729-733.

30. Choate K, Barbetti J, Currey J. Tracheostomy decannulation failure rate following critical illness: a prospective descriptive study. Aust Crit Care. 2009;22(1):8-15.

31. Hernández G, Ortiz R, Pedrosa A, Cuena R, Vaquero C, González P. La indicación de la traqueotomía condiciona las variables predictoras del tiempo hasta la decanulación en pacientes críticos. Med Intensiva. 2012.

32. Ricci Maccarini A, Stacchini M, Salsi D, Pieri F, Magnani M, Casolino D. Trans-tracheostomic endoscopy of the larynx in the evaluation of dysphagia. Acta Otorhinolaryngol Ital. 2007;27(6):290-293.

33. Donzelli J, Brady S, Wesling M, Craney M. Predictive value of accumulated oropharyngeal secretions for aspiration during video nasal endoscopic evaluation of the swallow. Ann Otol Rhinol Laryngol. 2003;112(5):469-475.

34. Farneti D. Valutazione videoendoscopica. In: Schindler O, Ruoppolo G, Schindler A, editors. Deglutologia. Turin: Edizioni Omega; 2001.

35. Cotton RT. Pediatric laryngotracheal stenosis. J Pediatr Surg. 1984;19(6):699-704.

36. Murgu S, Colt H. Tracheobronchomalacia and excessive dynamic airway collapse. Clin Chest Med. 2013;34(3):527-555.

37. Murgu SD, Colt HG. Description of a multidimensional classification system for patients with expiratory central airway collapse. Respirology. 2007;12(4):543-550.

38. Majid A, Fernández L, Fernández-Bussy S, Herth F, Ernst A. Tracheobronchomalacia. Arch Bronconeumol. 2010;46(4):196-202.

39. Rodrigues LB, Nunes TA. Importance of flexible bronchoscopy in decannulation of tracheostomy patients. Rev Col Bras Cir. 2015;42(2): 75-80.

40. Dunn PF, Goulet RL. Endotracheal tubes and airway appliances. Int Anesthesiol Clin. 2000;38(3):65-94. 
Clinical Audit is an international, peer-reviewed, open access journal focusing on the processes and outcomes of clinical audit in any area of healthcare. All aspects of patient care are addressed within the journal and practitioners from all disciplines are invited to submit their work. Areas covered include: Publication of audits; How an audit has changed practice;

Submit your manuscript here: https://www.dovepress.com/clinical-audit-journal
Dovepress

Practical tips on how to do audits and to avoid pitfalls; How audits have changed patient care; Calls and justifications for new audits. The manuscript management system is completely online and includes a very quick and fair peer-review system, which is all easy to use. Visit http://www.dovepress. com/testimonials.php to read real quotes from published authors. 